Finally, this case demonstrates the importance of timely and correct diagnosis in PAH. Evidence suggests that treatment of mildly symptomatic patients can be beneficial, and indeed data from the EARLY trial [5] led to regulatory authority approval for the use of bosentan in the treatment of $\mathrm{PAH}$ patients in NYHA/WHO FC II and a recommendation for treatment of such patients in recent guidelines [3]. In this case the patient's condition deteriorated between her initial misdiagnosis of post-partum depression and her referral to our specialist centre 3 months later, removing the option for early treatment before her symptoms became marked.

\section{O. Sitbon}

Université Paris Sud-11, Centre de Référence de l'Hypertension Pulmonaire Sévère, Service de Pneumologie, INSERM U999, Hôpital Antoine Béclère, Clamart, France.

Correspondence: O. Sitbon, Service de Pneumologie, Hôpital Antoine Béclère, 92141, Clamart, France. E-mail: olivier.sitbon@ abc.aphp.fr

Statement of Interest: O. Sitbon has relationships with drug companies including Actelion, Bayer-Schering, GSK, Lilly, Pfizer and United Therapeutics. In addition to being an investigator in trials involving these companies, relationships include consultancy service and membership of scientific advisory boards. He has received reimbursement for attending symposium and funds for research from Actelion, Pfizer, GSK, Lilly and Bayer-Schering. He has received fees for speaking from Actelion, Bayer-Schering, GSK, Lilly, Pfizer and United Therapeutics.

Provenance: Publication of this peer-reviewed article was supported by Actelion Pharmaceuticals Ltd, Switzerland (unrestricted grant, European Respiratory Review issue 118).

Acknowledgements: Editorial assistance was frovided by L. Thomas, Elements Communications Ltd (Westerham, UK), supported by Actelion Pharmaceuticals Ltd (Allschwil, Switzerland).

\section{REFERENCES}

1 Galiè N, Negro L, Simonneau G. The use of combination therapy in pulmonary arterial hypertension: new developments. Eur Respir Rev 2009; 18: 148-153.

2 Montani D, O'Callaghan DS, Jaiis X, et al. Implementing the ESC/ ERS pulmonary hypertension guidelines: real-life cases from a national referral centre. Eur Respir Rev 2009; 18: 272-290.

3 Galiè N, Hoeper MM, Humbert M, et al. Guidelines for the diagnosis and treatment of pulmonary hypertension. Eur Respir J 2009; 34: 1219-1263.

4 Barst RJ, Gibbs JS, Ghofrani HA, et al. Updated evidence-based treatment algorithm in pulmonary arterial hypertension. J Am Coll Cardiol 2009; 54: Suppl. 1, S78-S84.

5 Galiè N, Rubin LJ, Hoeper M, et al. Treatment of patients with mildly symptomatic pulmonary arterial hypertension with bosentan (EARLY study): a double-blind, randomised controlled trial. Lancet 2008; 371: 2093-2100.

\title{
Association of pulmonary aspergilloma and allergic bronchopulmonary aspergillosis
}

\section{To the Editor:}

We have read with interest recent reports on the complex interactions between Aspergillus fumigatus and the lungs [1-3]. A. fumigatus may be involved in different respiratory diseases including aspergilloma, allergic bronchopulmonary aspergillosis (ABPA) or invasive infectious manifestations. However, these different manifestations are usually not concomitantly present and there are only a few studies that report the association between these complications [4-14]. We wish to present a case of fully documented aspergilloma and ABPA in a patient with difficult asthma, which represented a difficult therapeutic challenge.

A 50-yr-old female, nonsmoker was followed up since 2000 for difficult to control non-atopic asthma associated with severe rhinosinusitis. In March 2008, the patient presented to the respiratory department (Hôpital Antonie Béclère, Clamart, France) with a 1-month history of mild recurrent haemoptysis. Physical examination was normal. Chest radiography showed excavated lung opacity in the superior left field. A thoracic computed tomography $(\mathrm{CT})$ scan revealed a cavity with a fungus ball in the left upper lobe and two other opacities in the left lower lobe (fig. 1). There was no fungus ball in the sinuses. Fiberoptic bronchoscopy showed no abnormality. Bronchial fluid aspiration and bronchoalveolar lavage stains and cultures did not detect either resistant acid alcohol bacilli or Aspergillus. The eosinophil count was $1.5 \times 10^{9} \cdot \mathrm{L}^{-1}$ and total serum immunoglobulin (Ig)E levels were elevated $\left(2,236 \mathrm{KU} \cdot \mathrm{L}^{-1}\right.$, normal range: 0 $\left.113 \mathrm{KU} \cdot \mathrm{L}^{-1}\right)$. A fumigatus specific $\mathrm{IgE}$ was positive $\left(41.2 \mathrm{KU} \cdot \mathrm{L}^{-1}\right)$. Aspergillus serology including Aspergillus immunoelectrophoresis was positive. Antinuclear antibodies and antineutrophil cytoplasmic antibodies were negative. A CT scan of the chest revealed persistence of the left upper lobe opacity, disappearance of the other opacities and localised bronchiectasis of the left lower lobe. No evidence of transient pulmonary infiltrates has been documented. A diagnosis of concomitant aspergilloma and ABPA was suspected. A wedge resection of the left upper lobe was performed (fig. 2). The histopathological examination of the resected wedge revealed an aspergilloma with fungal hyphae (fig. 3). Culture of this material yielded A. fumigatus. The patient received itraconazole post-operatively at a dose of $300 \mathrm{mg} \cdot$ day $^{-1}$ and was continued on inhaled corticosteroids. 5 months after the intervention, her total serum IgE count dropped to $1,769 \mathrm{KU} \cdot \mathrm{L}^{-1}$ and her A. fumigatus specific IgE level dropped to $33.8 \mathrm{KU} \cdot \mathrm{L}^{-1}$. 

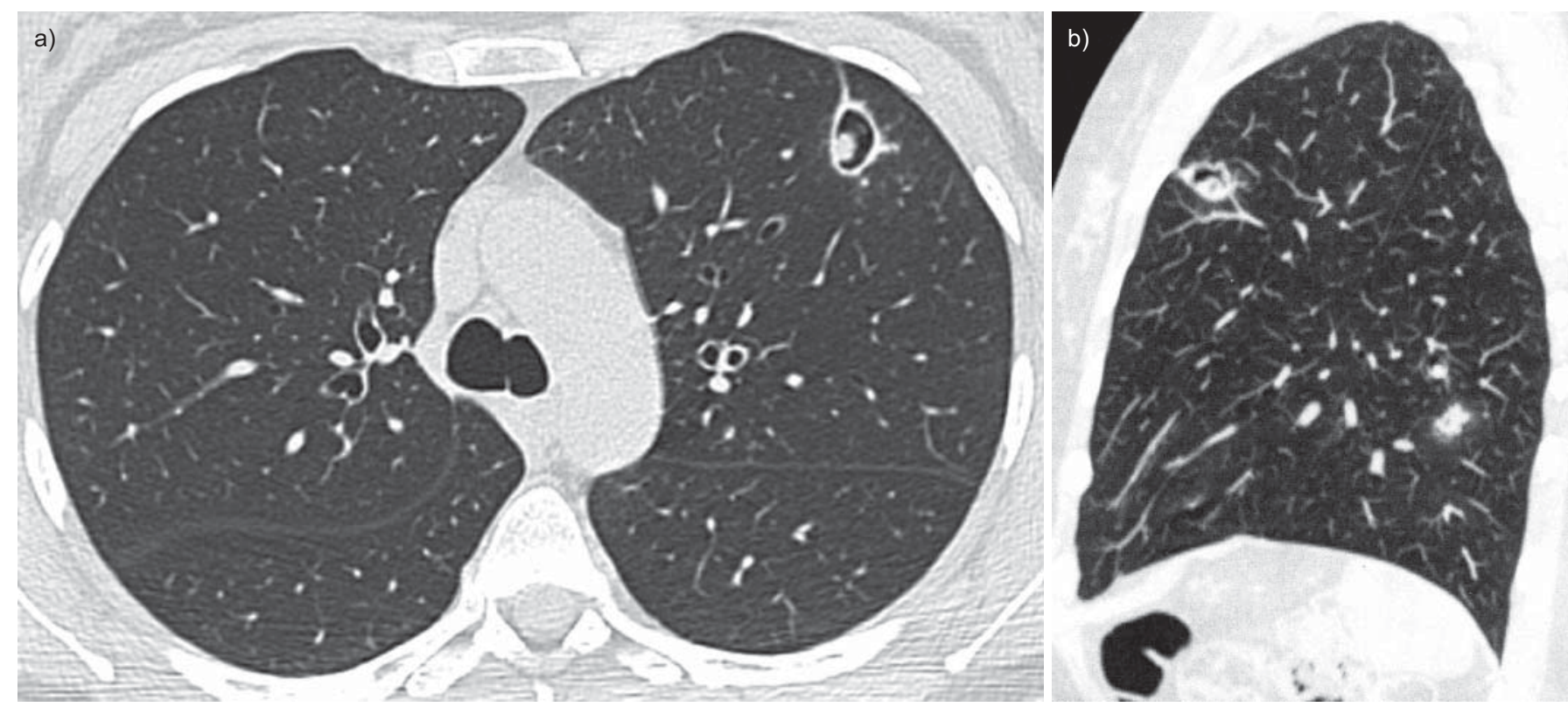

FIGURE 1. Thoracic computed tomography revealing a 16-mm cavity with a fungus ball in the anterior segment of the left upper lobe (a), and two other lesions of the left apical segment and the left lower lobe measuring $9 \mathrm{~mm}$ and $19 \mathrm{~mm}(\mathrm{~b})$, respectively.

2 yrs after surgery the patient remains asymptomatic with a normal thoracic CT apart from localised bronchiectasis of the left lower lobe. Itraconazole was discontinued after 18 months of treatment.

A. fumigatus may cause significant morbidity in the respiratory tract (aspergilloma, ABPA and invasive infectious manifestations). Classically, only one disease occurs in each patient. However, there are a few case reports of the association between these complications [2, 3, 9-11, 14, 15]. Aspergilloma is a fungus ball caused by saprophytic overgrowth of Aspergillus species in a cavitary or cystic lung disease. However, tissue necrosis and invasion of blood vessels does not occur. Aspergilloma is an unusual complication of ABPA [9]. The mechanism by which this occurs is by focal dilatation of the ectatic bronchi, due to accumulation of material

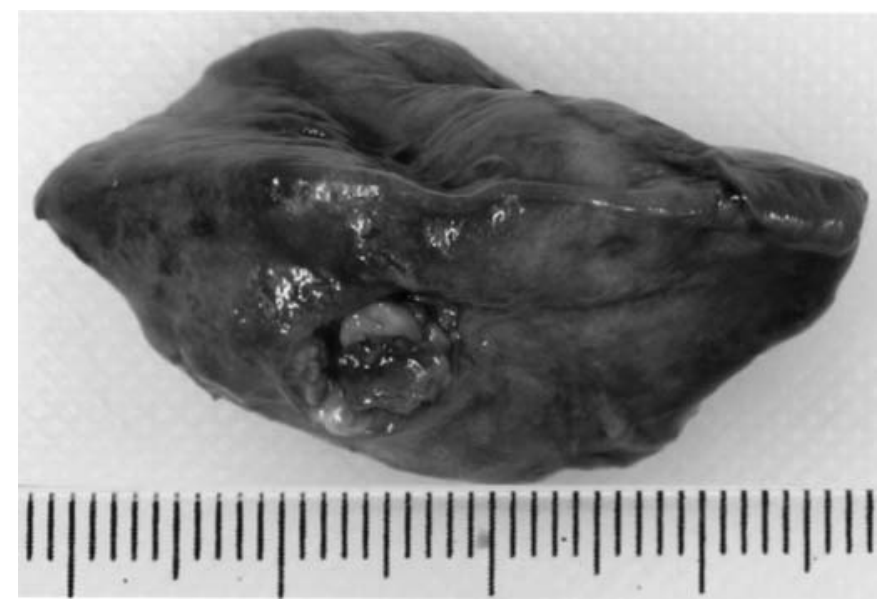

FIGURE 2. Macroscopic examination of the wedge resected lung revealing a $12 \mathrm{~mm}$ aspergilloma with a fungus ball. produced during the immune response to Aspergillus. The cavity can then be colonised by Aspergillus species to form an aspergilloma. In such cases aspergilloma formation is thought to be accelerated by corticosteroids [16].

In our patient, we made the diagnosis of concomitant aspergilloma and ABPA. As was observed in our patient, patients with ABPA often present with poorly controlled asthma and peripheral eosinophilia, and exhibit exacerbations followed by remissions when receiving systemic corticosteroid therapy. The association of ABPA and aspergilloma may present a real therapeutic challenge due to the central role of corticosteroids in the management of ABPA and the possible risk of aspergilloma growth with corticosteroid therapy. Only a few studies have demonstrated the role of intraconazole in improving asthma in ABPA regardless of corticosteroid therapy $[17,18]$. Medical treatment is unable to cure this condition and surgery remains the treatment of choice for aspergilloma became of the risk of unpredictable life-threatening haemoptysis. Our patient underwent a wedge resection as the rest of the parenchyma was normal. She received itraconazole and inhaled corticosteroids as she had superimposed ABPA, with a favourable outcome. This case highlights that the association of aspergilloma and $\mathrm{ABPA}$ is rare but possible.

\section{Montani ${ }^{*, \#, \pi}$, I. Zendah ${ }^{+}$, L. Achouh, ${ }^{*, \#, \pi}$, P. Dorfmuller, $*, \oplus, \Phi$, , O. Mercier ${ }^{\dagger}$, G. Garcia ${ }^{*, \#, q}$,

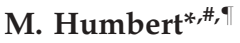

*Université Paris-Sud, Faculté de Médecine, Kremlin-Bicêtre, \#Assistance Publique, Hôpitaux de Paris, Service de Pneumologie et Réanimation Respiratoire, Hôpital Antoine Béclère, Clamart, "INSERM U999, Hypertension Artérielle Pulmonaire, Physiopathologie et Innovation Thérapeutique, Centre Chirurgical Marie-Lannelongue, ${ }^{\S}$ Service d'Anatonie et 

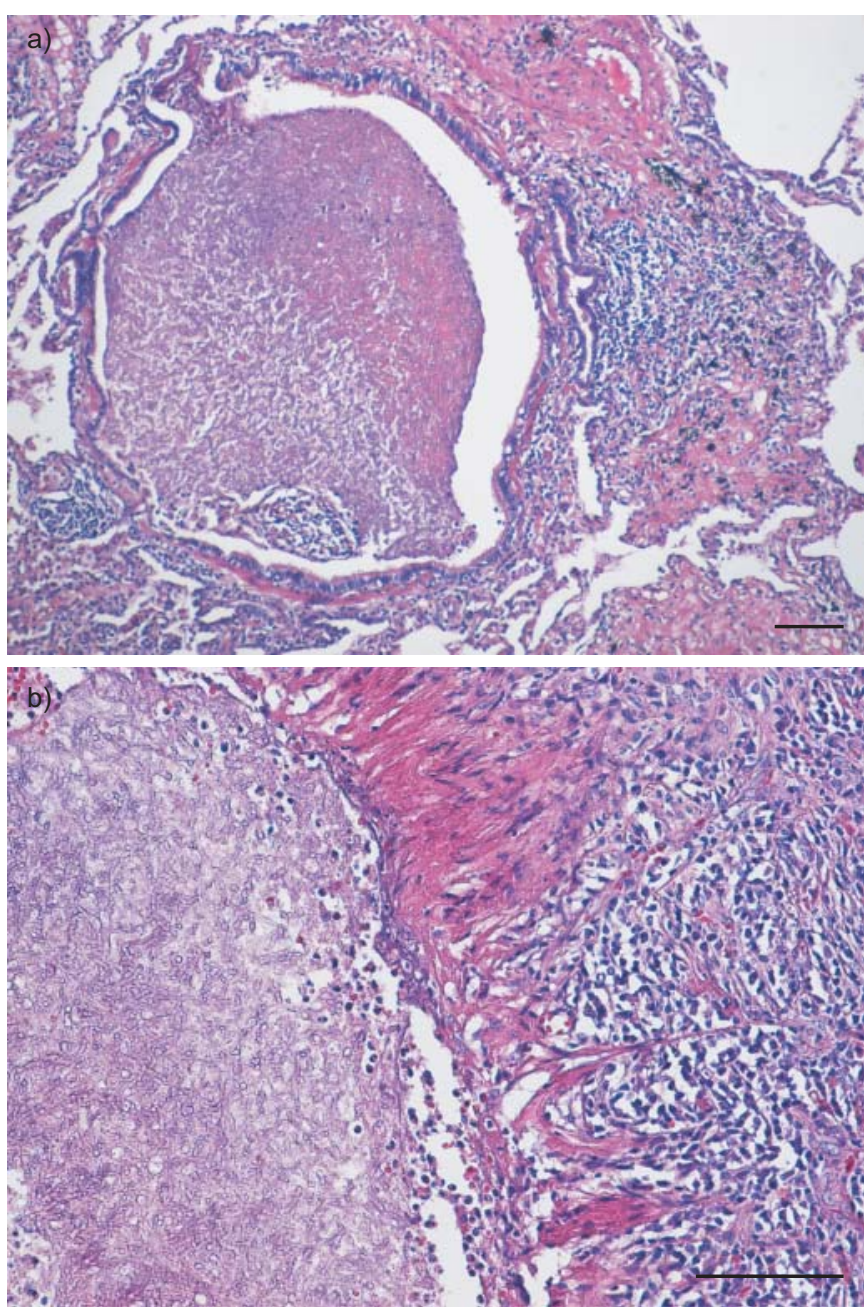

FIGURE 3. Histological examination (Haematoxylin-eosin staining) of the pulmonary aspergilloma. a) A dilated bronchus filled with necrosed aspergillus hyphae. The small airway is apparently connected to the main cavity (top left) b) Partially necrosed masses of hyphae within the cavity and the so-called fungus ball (left). Note the granulation tissue of the cavity wall, consisting of fibroblasts and inflammatory cells that are mainly lymphocytes, plasma cells and eosinophil leukocytes. Scale bars $=100 \mu \mathrm{m}$.

de Cytologie Pathologiques, Centre Chirurigal MarieLannelongue, ${ }^{f}$ Service de Chirurgie Thoracique, Centre Chirurgical Marie-Lannelongue, Le Plessis-Robinson, France. ${ }^{+}$Aderrahmen Mami Hospital of Respiratory Diseases, Dept I, Ariana, Tunisia.

Correspondence: D. Montani, Service de Pneumologie, Hôpital Antoine-Béclère, Assistance Publique, Hôpitaux de Paris, Université Paris-Sud 11, 157 rue de la Porte de Trivaux, 92140, Clamart, France. E-mail: david.montani@abc.aphp.fr

Statement of Interest: D. Montani has relationships with drug companies including $\mathrm{AB}$ Science, Actelion, Bayer Schering, GSK, Novartis, Pfizer and United Therapeutics. In addition to being an investigator in trials involving these companies, relationships include consultancy services and membership of scientific advisory boards. M. Humbert has relationships with drug companies including $\mathrm{AB}$ Science, Actelion, Bayer Schering, GSK, Novartis, Pfizer and United Therapeutics. In addition to being an investigator in trials involving these companies, relationships include consultancy services and membership of scientific advisory boards.

Provenance: Submitted article, peer reviewed.

\section{REFERENCES}

1 Agarwal R, Gupta D, Aggarwal AN, et al. Allergic bronchopulmonary aspergillosis: lessons from 126 patients attending a chest clinic in north India. Chest 2006; 130: 442-448.

2 Prasad R, Garg R, Dua R, et al. Concomitant allergic bronchopulmonary aspergillosis and allergic fungal sinusitis. Indian J Med Sci 2007; 61: 532-534.

3 Seville RA, Murchison J, Riha RL. An unusual cause of blindness. Eur Respir J 2010; 35: 216-217.

4 Safirstein BH, D'Souza MF, Simon G, et al. Five-year follow-up of allergic bronchopulmonary aspergillosis. Am Rev Respir Dis 1973, 108: 450-459.

5 Safirstein BH. Aspergilloma consequent to allergic bronchopulmonary aspergillosis. Am Rev Respir Dis 1973; 108: 940-943.

6 Ein M, Wallace RJ, Williams TJ. Allergic bronchopulmonary aspergillosis-like syndrome consequent to aspergilloma. Am Rev Respir Dis 1979; 119: 811-820.

7 Rosenberg I, Greenberger P. Allergic bronchopulmonary aspergillosis and aspergilloma. Long-term follow-up without enlargement of a large multiloculated cavity. Chest 1984; 85: 123-125.

8 Shah A, Khan ZU, Chaturvedi S, et al. Allergic bronchopulmonary aspergillosis with coexistent aspergilloma: a long-term followup. J Asthma 1989; 26: 109-115.

9 Reich J. Pneumothorax due to pleural perforation of a pseudocavity containing aspergillomas in a patient with allergic bronchopulmonary aspergillosis. Chest 1992; 102: 652-653.

10 Bhagat R, Shah A, Jaggi O, et al. Concomitant allergic bronchopulmonary aspergillosis and allergic Aspergillus sinusitis with an operated aspergilloma. J Allergy Clin Immunol 1993; 91: 1094-1096.

11 Shah A, Bhagat R, Panchal N, et al. Allergic bronchopulmonary aspergillosis with middle lobe syndrome and allergic Aspergillus sinusitis. Eur Respir J 1993; 6: 917-918.

12 Agarwal A, Bhagat R, Panchal N, et al. Allergic bronchopulmonary aspergillosis with aspergilloma mimicking fibrocavitary pulmonary tuberculosis. Asian Pac J Allergy Immunol 1996; 14: 5-8.

13 Sharma P, Agarwal AK, Shah A. Formation of an aspergilloma in a patient with allergic bronchopulmonary aspergillosis on corticosteroid therapy. Indian J Chest Dis Allied Sci 1998; 40: 269-273.

14 Marcq M, Germaud P, Cellerin L, et al. [Complex respiratory aspergillosis: diagnostic and therapeuthic difficulties]. Rev Mal Respir 2004; 21: 1162-1166.

15 Shah A, Panjabi C. Contemporaneous occurrence of allergic bronchopulmonary aspergillosis, allergic Aspergillus sinusitis, and aspergilloma. Ann Allergy Asthma Immunol 2006; 96: 874-878.

16 Akimoto T, Saito O, Inoue M, et al. Rapid formation of Aspergillus mycetoma in a patient receiving corticosteroid treatment. Serial radiographic observation over two months. Intern Med 2007; 46 : 733-737.

17 Salez F, Brichet A, Desurmont S, et al. Effects of itraconazole therapy in allergic bronchopulmonary aspergillosis. Chest 1999; 116: $1665-1668$

18 Stevens DA, Schwartz HJ, Lee JY, et al. A randomized trial of itraconazole in allergic bronchopulmonary aspergillosis. $N$ Engl J Med 2000; 342: 756-762. 